\title{
CARACTERIZAÇÃO DE LIXIVIADO DE ATERRO SANITÁRIO E PROPOSTAS DE TRATAMENTO
}

\author{
CHARACTERIZATION OF LANDFILL LEACHATE AND TREATMENT \\ PROPOSALS
}
Ana Paula Jambers Scandelai ${ }^{1}$; Danielly Cruz Campos Martins ${ }^{1}$; Camila Dias Pinaffi ${ }^{2}$; Célia Regina Granhen Tavares ${ }^{1}$
${ }^{1}$ Universidade Estadual de Maringá, Departamento de Engenharia Química

E-mail: paulascandelai@hotmail.com; daniellyccampos@gmail.com; celia@deq.uem.br

\author{
${ }^{2}$ Universidade do Oeste Paulista - UNOESTE, Departamento de Solos e \\ Nutrição de Plantas \\ e-mail: camila pinaffi@hotmail.com
}

RESUMO - O lixiviado de aterro sanitário é constituído de diversas substâncias de difícil degradação. Este estudo objetivou avaliar o comportamento sazonal do lixiviado do aterro sanitário de Maringá-PR. $\mathrm{O}$ mesmo foi coletado em quatro estações do ano e caracterizado pelos parâmetros de $\mathrm{pH}, \mathrm{CE}$, cor, turbidez, $\mathrm{DQO}, \mathrm{DBO}, \mathrm{COT}, \mathrm{N}-\mathrm{NH}_{3}, \mathrm{~N}-\mathrm{NO}_{2}{ }^{-}, \mathrm{N}-$ $\mathrm{NO}_{3}{ }^{-}, \mathrm{ST}, \mathrm{STD}$ e SST. O lixiviado variou significativamente ao longo do ano, apresentando os parâmetros mais concentrados no verão, provavelmente devido à estação anterior, a qual apresentou maior temperatura e menor pluviosidade. A variabilidade e complexidade do efluente evidenciaram a sua necessidade de tratamento antes do seu lançamento. Diversas técnicas de tratamento foram propostas, considerando a inexistência de um processo universal para degradação de lixiviados; no entanto, a escolha da melhor combinação depende de diversos fatores inerentes ao lixiviado, clima, financeiros e legais.

Palavras-chave: matéria orgânica; compostos nitrogenados; variação sazonal.

Recebido em: 10/08/2018 Revisado em: 22/08/2018 Aprovado em: 27/08/2018
ABSTRACT - The landfill leachate is constituted by several substances that of difficult degradation. This study aimed to evaluate the leachate seasonal behavior at Maringá-PR landfill. The landfill leachate was collected in four seasons of the year and characterized by $\mathrm{pH}, \mathrm{EC}$, color, turbidity, COD, BOD, TOC, $\mathrm{NH}_{3}-\mathrm{N}, \mathrm{NO}_{2}^{-}-\mathrm{N}, \mathrm{NO}_{3}-\mathrm{N}, \mathrm{TS}$, TDS and TSS parameters. The leachate varied significantly over the year, presenting the most concentrated parameters in the summer, probably due to the previous season, which presented higher temperature and lower rainfall. The variability and complexity of the leachate evidenced its need for treatment before its discharge. Several treatment techniques were proposed, considering the inexistence of a universal process for 
the degradation of landfills leachates; however, the choice of the best combination depends on several factors inherent to leachate, climate, financial and legal.

Keywords: organic matter; nitrogen compounds; seasonal variation. 


\section{INTRODUÇÃO}

No Brasil, dentre as opções de destinação de resíduos sólidos urbanos (RSU), a disposição em aterros sanitários é a prática mais comum (ASSOCIAÇÃO BRASILEIRA DE EMPRESAS DE LIMPEZA PÚBLICA E RESÍDUOS ESPECIAIS, 2017).

Nos aterros sanitários, os RSU sofrem uma série de transformações físico-químicas e biológicas, levando à produção do efluente denominado lixiviado. A quantidade e qualidade do lixiviado gerado dependem de diversos fatores, referentes aos resíduos (composição, densidade e teor de umidade inicial), ao clima (precipitação, escoamento superficial, temperatura e estações do ano), e ao sistema operacional (profundidade e idade do aterro e nível de compactação das camadas de RSU) (LISK, 1991).

Apesar da variabilidade dos lixiviados, os mesmos apresentam, em sua composição, altas concentrações de matéria inorgânica e orgânica, biodegradável e recalcitrante, metais tóxicos, compostos nitrogenados, entre outros contaminantes, além de elevada coloração e odor desagradável (CHRISTENSEN et al., 2001). Em vista de seu potencial risco poluidor, esse efluente deve ser tratado antes de lançado nos corpos hídricos receptores, visando atender aos padrões de lançamento estabelecidos pelas legislações brasileiras, como as Resoluções CONAMA n. 357/2005 e n. 430/2011 (BRASIL, 2005, 2011) e Resolução n. 070/2009 (PARANÁ, 2009), de forma a promover a segurança sanitária e a preservação do ambiente.

Com base nisso, o presente estudo visou caracterizar e avaliar o comportamento do lixiviado do aterro sanitário de Maringá$P R$, a fim de se obter a variabilidade anual de sua composição.

\section{MÉTODOS}

O lixiviado foi coletado no reservatório de lixiviado do aterro sanitário de Maringá, Paraná, Brasil.
A fim de avaliar o comportamento do lixiviado em função das características climáticas e conhecer a sua variabilidade ao longo do tempo, foram realizadas quatro coletas no ano de 2017, durante as quatro estações do ano: verão, outono, inverno e primavera.

Para a caracterização do lixiviado, foram analisados os parâmetros de $\mathrm{pH}$, condutividade elétrica (CE) e turbidez, de acordo com as metodologias dos equipamentos ( $\mathrm{pH}$-metro Digimed $\mathrm{DM} / 20$, medidor multiparâmetro Hanna HI4522 e turbidímetro Digimed DM-TU, respectivamente), cor aparente e verdadeira (platina-cobalto - método 8025) (HACH COMPANY, 1996), demanda química de oxigênio (DQO) (colorimetria - método 5220D), demanda bioquímica de oxigênio (DBO) (respirometria - método 5210B) e carbono orgânico total (COT) (combustão a alta temperatura, em um analisador Shimadzu TOC-L CSH - método 5310B), de acordo com as metodologias propostas no Standard Methods of Water and Wastewater (AMERICAN PUBLIC HEALTH ASSOCIATION, 1998). A série nitrogenada foi quantificada pelos parâmetros de nitrogênio amoniacal total $\left(\mathrm{N}-\mathrm{NH}_{3}\right)$ (potenciometria, com eletrodo Thermo Scientific Orion 9512BNWP e medidor multiparâmetro Thermo Scientific Orion Star 4), nitrito $\left(\mathrm{N}-\mathrm{NO}_{2}{ }^{-}\right)$e nitrato (N$\mathrm{NO}_{3}{ }^{-}$) (redução com sulfato ferroso - método 8153 e redução por cádmio - método 8039 , respectivamente) (HACH COMPANY, 1996). Por fim, foi quantificada a série de sólidos: sólidos totais (ST), sólidos dissolvidos totais (SDT) e sólidos suspensos totais (SST) por gravimetria (métodos 2540B, 2540C e 2540D, respectivamente) (AMERICAN PUBLIC HEALTH ASSOCIATION, 1998). Tais parâmetros são de extrema importância para a caracterização de efluentes e, além disso, são parâmetros das legislações ambientais, as quais apresentam limites máximos permitidos para o lançamento dos mesmos. 


\section{RESULTADOS E DISCUSSÕES}

O aterro sanitário de Maringá-PR, localizado no município de Maringá, possui uma área de $290.400 \mathrm{~m}^{2}$ e está em operação desde 2010. O mesmo recebe, aproximadamente, $40 \mathrm{t} \mathrm{dia}^{-1}$ de resíduos sólidos não perigosos de grandes geradores (estabelecimentos que geram $50 \mathrm{~kg} \mathrm{dia}^{-1}$ ou $100 \mathrm{~L} \mathrm{dia}^{-1}$ de resíduos) e $360 \mathrm{t} \mathrm{dia}^{-1}$ de RSU e de varrição. $O$ aterro gera, diariamente, um volume aproximado de $30 \mathrm{mil}$ litros de lixiviado (MARTINS, 2017), o qual apresenta características distintas conforme estação do ano, como pode ser observada na Tabela 1.

Diferentemente dos demais parâmetros analisados, independentemente da estação do ano, o lixiviado gerado no aterro de Maringá apresentou o pH próximo da neutralidade e dentro do limite de lançamento estabelecido pela Resolução CONAMA n. 430/2011 (BRASIL, 2011).

A CE, a qual está diretamente relacionada à presença de compostos dissolvidos, em especial ânions e cátions dissociados, indica a facilidade com a qual a água transmite corrente elétrica, como abordado por Lacerda et al. (2015). Embora não seja um parâmetro presente na legislação para lançamento de efluentes, a CE é considerada um indicador importante da concentração de íons. No lixiviado em estudo, a CE se apresentou elevada em todas as estações, o que pode, segundo Thomas et al. (2009), causar efeito tóxico ao efluente.

Tabela 1. Características físico-químicas do lixiviado gerado no aterro sanitário de Maringá-PR em diferentes estações.

\begin{tabular}{lccccc}
\hline \multirow{2}{*}{ Parâmetro } & \multicolumn{4}{c}{ Estações do ano } & Limite de \\
\cline { 2 - 5 } & Verão & Outono & Inverno & Primavera & Lançamento \\
\hline $\mathrm{pH}$ & 7,5 & 7,7 & 8,3 & 7,6 & $5,0-9,0^{\mathrm{a}}$ \\
$\mathrm{CE}\left(\mu \mathrm{S} \mathrm{cm}^{-1}\right)$ & 11720 & 10270 & 10160 & 8004 & $\mathrm{ND}$ \\
Cor Aparente $(\mathrm{uH})$ & 4260 & 3820 & 936 & 2490 & $\mathrm{ND}$ \\
Cor Verdadeira (uH) & 3060 & 1060 & 644 & 1495 & $75^{\mathrm{b}}$ \\
Turbidez (UNT) & 290 & 114 & 85 & 109 & $100^{\mathrm{b}}$ \\
$\mathrm{DQO}\left(\mathrm{mg} \mathrm{L}^{-1}\right)$ & 3526 & 2265 & 1048 & 1076 & $200^{\mathrm{c}}$ \\
$\mathrm{DBO}\left(\mathrm{mg} \mathrm{L}^{-1}\right)$ & 992 & 442 & 165 & 219 & $50^{\mathrm{c}}$ \\
$\mathrm{DBO} / \mathrm{DQO}^{-1}$ & 0,28 & 0,19 & 0,16 & 0,20 & $\mathrm{ND}$ \\
$\mathrm{COT}\left(\mathrm{mg} \mathrm{L}^{-1}\right)$ & 1190 & 646 & 334 & 352 & $\mathrm{ND}$ \\
$\left.\mathrm{N}-\mathrm{NH} \mathrm{mg} \mathrm{L}^{-1}\right)$ & 632 & 410 & 505 & 496 & $20^{\mathrm{b}}$ \\
$\mathrm{N}-\mathrm{NO}_{2}^{-}\left(\mathrm{mg} \mathrm{L}^{-1}\right)$ & 40 & 27 & 30 & 5 & $1,0^{\mathrm{b}}$ \\
$\mathrm{N}-\mathrm{NO}_{3}^{-}\left(\mathrm{mg} \mathrm{L}^{-1}\right)$ & 4 & 32 & 8 & 6 & $10^{\mathrm{b}}$ \\
$\mathrm{ST}\left(\mathrm{mg} \mathrm{L}^{-1}\right)$ & 8650 & 5568 & 6941 & $\mathrm{ND}$ & $\mathrm{ND}$ \\
$\mathrm{SDT}\left(\mathrm{mg} \mathrm{L}^{-1}\right)$ & 8516 & 5410 & 6801 & $\mathrm{ND}$ & $500^{\mathrm{b}}$ \\
$\mathrm{SST}\left(\mathrm{mg} \mathrm{L}^{-1}\right)$ & 134 & 158 & 141 & $\mathrm{ND}$ & $\mathrm{ND}$ \\
\hline
\end{tabular}

Notas: ND = parâmetro não determinado; (1) Limites determinados pelas Resoluções (a) CONAMA n. 430/2011 (BRASIL, 2011); (b) CONAMA n. 357/2005 (BRASIL, 2005); (c) CEMA n. 070/2009 (PARANÁ, 2009).

Fonte: os autores.

Com relação ao parâmetro de cor, tanto a cor aparente quanto a verdadeira apresentaram elevadas concentrações em todas as estações do ano, mostrando-se acima do estabelecido pela legislação. A cor dos lixiviados é ocasionada pelos sólidos dissolvidos, em especial pela presença de substâncias húmicas, provenientes da degradação de matéria orgânica, as quais conferem a coloração marrom, peculiar a esse efluente.

A turbidez, por sua vez, ocasionada pela presença de partículas coloidais suspensas, apresentou-se dentro do padrão 
estabelecido pela Resolução CONAMA $\mathrm{n}$. 357/2005 (BRASIL, 2005) apenas no inverno; próximo ao limite no outono e na primavera; e excedendo bastante esse limite no verão. Tal fato pode estar relacionado com a concentração de chuvas nesse período.

Os lixiviados analisados apresentaram elevada concentração de matéria orgânica, representada pelos parâmetros indiretos de DBO e DQO e pelo parâmetro direto de COT, estando os dois primeiros sempre acima do limite de $50 \mathrm{mg} \mathrm{L}^{-1}$ e $200 \mathrm{mg} \mathrm{L}^{-1}$, os quais são estabelecidos como máximos pela Resolução CEMA n. 070/2009 (PARANÁ, 2009). Além disso, em vista da razão DBO/DQO menor que 0,5 , os lixiviados apresentaram característica de baixa biodegradabilidade, indicando, segundo Renou et al. (2008), a presença majoritária de substâncias orgânicas recalcitrantes, o que dificulta seu processo de degradação.

Com relação aos compostos nitrogenados analisados, a amônia $\left(\mathrm{N}-\mathrm{NH}_{3}\right)$ é a forma predominante encontrada nos lixiviados, seguida de nitrito $\left(\mathrm{N}-\mathrm{NO}_{2}{ }^{-}\right)$e nitrato $\left(\mathrm{N}-\mathrm{NO}_{3}{ }^{-}\right)$. Dentre as três formas, o nitrato foi o único parâmetro que se adequou ao limite de lançamento no verão, inverno e primavera; no entanto, se excedeu no outono. De acordo com Von Sperling (2014), enquanto a amônia é proveniente da decomposição de compostos orgânicos nitrogenados, o nitrito e o nitrato são produtos resultantes de sua oxidação. Esses compostos, se não removidos do efluente, podem contribuir com intoxicação do meio aquático e com a eutrofização de corpos receptores.

A série de sólidos analisadas evidenciou a predominância, em todos os casos, de sólidos dissolvidos em detrimento dos sólidos suspensos, o que corroborou com a elevada coloração dos efluentes e menores concentrações de turbidez, respectivamente.

A variabilidade das características do lixiviado está intrinsicamente relacionada com o clima, precipitação e temperatura das estações do ano analisadas. O clima do município de Maringá é definido, segundo a classificação climática Köppen, como subtropical (Cfa), com verão e inverno definidos e com temperaturas médias nos meses mais frio e mais quente, respectivamente, inferior a $18{ }^{\circ} \mathrm{C}$ e superior a $22{ }^{\circ} \mathrm{C}$. Nessa região, a estação seca não é definida, ocorrendo precipitação em todos os meses do ano. No entanto, os verões são quentes e com concentração das chuvas. (INSTITUTO AGRONÔMICO DO PARANÁ, 2018). No ano de 2017, a precipitação anual acumulada no município foi de $2048,4 \mathrm{~mm}$, segundo dados do Instituto Nacional de Meteorologia (2018). Na Tabela 2 são apresentados os dados de precipitação e temperaturas máximas e mínimas, ocorridas nos meses de coleta de lixiviado.

De acordo com a Tabela 2, observa-se que o mês de julho foi o mais seco, visto a sua menor precipitação acumulada, enquanto que setembro foi o mês mais quente, apresentando maior temperatura máxima. Dezembro, ao contrário, foi o mês de maior precipitação dentre aqueles de coleta; comportamento este caracterizado pela estação chuvosa (verão) no município.

Correlacionando os dados das Tabelas 1 e 2, observa-se que as características mais poluentes do lixiviado ocorreram no verão (Tabela 1), o qual apresentou maiores concentrações de todos os parâmetros avaliados, com exceção ao nitrato e aos SST. Apesar de o verão se tratar de uma estação chuvosa (Tabela 2) que, teoricamente, diluiria o lixiviado, deixando-o com características mais amenas, a elevação de tais parâmetros é justificada. Isso porque, tanto a água pluvial infiltrada durante as chuvas, quanto a contida nos próprios resíduos (sobretudo orgânicos), as quais formam o lixiviado, levam certo tempo desde a infiltração, chegada à rede coletora até o tanque de armazenamento do lixiviado. 
Tabela 2. Dados de precipitação total e temperaturas médias registradas no município de Maringá-PR, nos meses de coleta de lixiviado.

\begin{tabular}{ccccc}
\cline { 2 - 5 } & \multicolumn{4}{c}{ Período de coleta } \\
\cline { 2 - 5 } & $\begin{array}{c}\text { Outono } \\
\text { (Jun/17) }\end{array}$ & $\begin{array}{c}\text { Inverno } \\
\text { (Jul/17) }\end{array}$ & $\begin{array}{c}\text { Primavera } \\
\text { (Set/17) }\end{array}$ & $\begin{array}{c}\text { Verão } \\
\text { (Dez/17) }\end{array}$ \\
\hline Precipitação Total $(\mathrm{mm})$ & 103 & 0,0 & 33,2 & 210,1 \\
Temperatura Máxima Média $\left({ }^{\circ} \mathrm{C}\right)$ & 23,7 & 24,9 & 32,5 & 29,7 \\
Temperatura Mínima Média $\left({ }^{\circ} \mathrm{C}\right)$ & 14,8 & 14,3 & 20,9 & 21,5 \\
\hline
\end{tabular}

Fonte: Instituto Nacional de Meteorologia (2018).

Portanto, as elevadas concentrações dos poluentes do lixiviado, observadas no verão, podem ser decorrentes da estação anterior, primavera, a qual apresentou, dentre os meses analisados, maior temperatura máxima e baixa pluviosidade. Além disso, essa intensificação de parâmetros no verão pode ter ocorrido devido à coleta ter sido realizada em um mês de férias e festividades, o que acarreta em maiores consumo e geração de RSU e, consequentemente, maiores concentrações dos parâmetros do lixiviado.

Uma analogia pode ser feita para a estação do inverno, a qual apresentou um lixiviado com as menores concentrações de cor (aparente e verdadeira), turbidez e matéria orgânica (DQO, DBO e COT). Tais características podem ser devido às propriedades do lixiviado gerado durante o mês de coleta (julho), em que não ocorreram chuvas, e aos anteriores a este período, uma vez que junho apresentou pluviosidade moderada.

Diante das características do lixiviado (Tabela 1), verifica-se que este efluente apresentou grande variabilidade ao longo do ano, dentro de em um mesmo aterro. Estes dados evidenciam a necessidade do tratamento do lixiviado, de forma que haja uma degradação eficiente do mesmo, considerando as suas características mais extremas. Em virtude disso, a seleção do melhor método para o seu tratamento é complexa, uma vez que não existe um processo único e padronizado que permita a sua degradação em níveis aceitáveis de lançamento.
Diversos são os métodos e combinações utilizadas para o tratamento de lixiviados de aterros sanitários. A escolha dos processos com combinação ideal irá depender, dentre outros fatores, das características e quantidade do lixiviado gerado, dos requisitos legais, da eficiência de degradação dos poluentes promovida pelas tecnologias, bem como do custo de implantação e operação dos mesmos.

Dentre as técnicas disponíveis, têm-se os processos físico-químicos, biológicos e oxidativos avançados. Os processos físicoquímicos (como coagulação-floculaçãosedimentação, remoção de amônia por arraste de ar, precipitação química, adsorção e separação por membranas) são indicados para tratamento de lixiviados com matéria orgânica predominantemente refratária e pouco biodegradável, representada por baixos valores da razão DBO/DQO, como os lixiviados de aterros sanitários mais antigos, que também podem apresentar altas concentrações de amônia (KURNIAWAN et al., 2006; RENOU et al., 2008).

Os processos biológicos aeróbios (como lodos ativados, lagoas aeradas e filtros biológicos) e anaeróbios (como lagoas anaeróbias, reator anaeróbio de fluxo ascendente (UASB) e filtro anaeróbio) são indicados para os lixiviados jovens, os quais apresentam disponibilidade de compostos orgânicos biodegradáveis (DBO/DQO $>0,5$ ) e baixas concentrações de $\mathrm{N}-\mathrm{NH}_{3}$. No entanto, Kurniawan et al. (2010) destaca que nenhum dos tratamentos biológicos são universalmente aplicáveis para remoção de contaminantes de lixiviados, sobretudo recalcitrantes. 
Para lixiviados com baixa biodegradabilidade e altas concentrações de compostos refratários, os processos oxidativos avançados (POAs) são indicados para atuarem como pré-tratamento ao processo biológico ou pós-tratamento para polimento do efluente (OULEGO et al., 2016). Dentre os POAs mais comuns ao tratamento de lixiviado de aterro sanitário, destacam-se os que envolvem ozônio, radiação ultravioleta, peróxido de hidrogênio e catalisadores, combinados ou não entre si. Outra técnica oxidativa, recente e promissora, é a oxidação em água supercrítica (OASc), a qual possibilita elevada degradação dos poluentes de lixiviados, em reatores de pequeno porte e curto tempo reacional, como verificado nos trabalhos de Martins (2017) e Scandelai et al. (2018).

\section{CONSIDERAÇÕES FINAIS}

Todas as características aqui abordadas evidenciam a necessidade de se realizar o tratamento do lixiviado gerado no aterro sanitário de Maringá-PR, anteriormente ao seu lançamento em corpos receptores, a fim de garantir a segurança das águas e organismos que entram em contato com ela.

A caracterização do lixiviado nas quatro estações do ano permitiu confirmar a sua variabilidade e, consequentemente, sua complexidade de tratamento. Diante das diversas técnicas de tratamento disponíveis, deve ser realizada a escolha da combinação ideal, com base nas características do efluente e nos recursos disponíveis, uma vez que apenas uma técnica jamais será totalmente eficiente para a despoluição de lixiviados.

\section{AGRADECIMENTOS}

Os autores agradecem à CAPES.

\section{REFERÊNCIAS}

ASSOCIAÇÃO BRASILEIRA DE EMPRESAS DE LIMPEZA PÚBLICA E RESÍDUOS ESPECIAIS ABRELPE. Panorama de resíduos sólidos no Brasil - 2016. São Paulo: ABRELPE, 2017.

AMERICAN PUBLIC HEALTH ASSOCIATION APHA. Standard Methods for Examination of Water and Wastewater. 20. ed. Washington: APHA, 1998.

BRASIL. Ministério do Meio Ambiente. Conselho Nacional de Meio Ambiente CONAMA. Resolução $n^{\circ}$ 357, de 17 de março de 2005. Diário Oficial [da] República Federativa do Brasil, Poder Executivo, Brasília, DF, DOU nº 053, 18 mar. 2005. p. 5863.

BRASIL. Ministério do Meio Ambiente. Conselho Nacional de Meio Ambiente CONAMA. Resolução $n^{\circ}$ 430, de 13 de maio de 2011. Diário Oficial [da] República Federativa do Brasil, Poder Executivo, Brasília, DF, DOU n 92, 16 maio 2011. p. 89.

CHRISTENSEN, T. H. et al. Biogeochemistry of landfill leachate plumes. Applied Geochemistry, v. 16, p. 659-718, 2001. https://doi.org/10.1016/S0883-

2927(00)00082-2

$\mathrm{HACH}$ COMPANY. Procedures Manual of Spectrophotometer DR/2010. USA: Hach, 1996.

INSTITUTO AGRONÔMICO DO PARANÁ IAPAR. Cartas Climáticas do Paraná: classificação climática. Disponível em: http://www.iapar.br/pagina-863.html.

Acesso em: 08 maio 2018.

INSTITUTO NACIONAL DE METEOROLOGIA INMET. BDMEP - Banco de Dados Meteorológicos para Ensino e Pesquisa. Disponível em: http://www.inmet.gov.br/portal/index.php?r =bdmep/bdmep. Acesso em: 08 maio 2018 . 
KURNIAWAN, T. A. et al. Physico-chemical treatments for removal of recalcitrant contaminants from landfill leachate. Journal of Hazardous Materials, v. 129 , p. $80-100$, 2006.

https://doi.org/10.1016/j.jhazmat.2005.08.0

$\underline{10}$

KURNIAWAN, T. A. et al. Biological processes for treatment of landfill leachate. Journal of Environmental Monitoring, v. 12, p. 20322047, 2010. https://doi.org/10.1039/c0em00076k

LACERDA, A. B. et al. A condutividade da água como indicador de eficiência do processo de eletrodiálise. In: SEMINÁRIO DE PÓSGRADUAÇÃO (INOVAMUNDI), 8., 2015, Novo Hamburgo. Anais [...] Novo Hambugo: Universidade Feevale, 2015. p. 4-8.

LISK, D. J. Environmental efects of landfills. The Science of the Total Environment, v. 100, p. 415-468, 1991. https://doi.org/10.1016/00489697(91)90387-T

MARTINS, D. C. C. Tratamento de solução modelo de ácido húmico e lixiviado de aterro sanitário via oxidação em água supercrítica. 2017. Dissertação (Mestrado) Universidade Estadual de Maringá, Maringá, 2017.

OULEGO, P. et al. Impact of leachate composition on the advanced oxidation treatment. Water Research, v. 88, p. 389402, 2016. https://doi.org/10.1016/i.watres.2015.09.04 $\underline{8}$

PARANÁ (Estado). Conselho Estadual de Meio Ambiente - CEMA. Secretaria de Estado do Meio Ambiente e Recursos Hídricos - SEMA. Resolução $n^{\circ}$ 070, de 01 de outubro de 2009. Diário Oficial [da] República Federativa do Brasil, Poder Executivo, Brasília, DF, DOU n 8068, 01 out. 2009.
RENOU, S. et al. Landfill leachate treatment: Review and opportunity. Journal of Hazardous Materials, v. 150, p. 468-493, 2008.

https://doi.org/10.1016/i.jhazmat.2007.09.0 $\underline{77}$

SCANDELAI, A. P. J. et al. Combined processes of ozonation and supercritical water oxidation for landfill leachate degradation. Waste Management, v. 77, p. 466-476, 2018.

https://doi.org/10.1016/i.wasman.2018.04.0 $\underline{31}$

THOMAS, D. J. L. et al. Bioassays for the evaluation of landfill leachate toxicity. Journal of Toxicology and Environmental Health, Part B: Critical Reviews, v. 12, p. 83105, 2009. https://doi.org/10.1080/1093740080254529 $\underline{2}$

VON SPERLING, M. Introdução à qualidade das águas e o tratamento de esgotos. 4. ed. Belo Horizonte: Ed. UFMG, 2014. 